\title{
Measurement of Optic Nerve Sheath Diameter to Detect Increased Intracranial Pressure in Hypertensive Patients
}

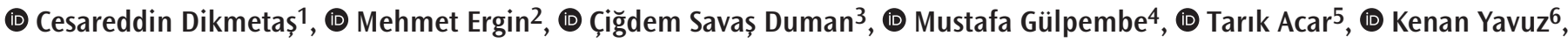 \\ (1) Başar Cander ${ }^{1}$, (1) Sadık Girișgin ${ }^{7}$, (1) Sedat Koçak ${ }^{7}$
}

${ }^{1}$ Clinic of Emergency Medicine, Kanuni Sultan Süleyman Training and Research Hospital, İstanbul Health Sciences University, İstanbul, Turkey 2Department of Emergency Medicine, Yıldırım Beyazıt University Faculty of Medicine, Ankara, Turkey

${ }^{3}$ Konya Provincial Health Directorate, Konya, Turkey

${ }^{4}$ Clinic of Emergency Medicine, Konya Training and Research Hospital, Konya, Turkey

${ }^{5}$ Clinic of Emergency Medicine, Beyhekim State Hospital, Konya, Turkey

${ }^{6}$ Clinic of Emergency Medicine, Rize State Hospital, Rize, Turkey

${ }^{7}$ Department of Emegergency Medicine, Necmettin Erbakan University Faculty of Medicine, Konya, Turkey

\begin{abstract}
Aim: Our aim was to measure optic nerve sheath diameter (ONSDM) by ultrasonography and to detect whether ONSDM reflected intracranial pressure in hypertensive patients.

Materials and Methods: This observational study was performed prospectively in 149 individuals, 77 (51.7\%) of whom were female, including 54 hypertensive-symptomatic patients, 45 hypertensive-asymptomatic patients, and 50 healthy volunteers referred to Emergency Medicine Service of Necmettin Erbakan University, Faculty of Medicine. Blood pressure was measured on the right and left upper extremities by the same sphygmomanometer following at least five minutes of rest. ONSDM was recorded. All measurements of hypertensive-symptomatic patients were repeated at 30th minute following antihypertensive therapy.

Results: Moderate-to-well statistically significant correlations were determined between initial mean systolic blood pressure and ONSDM ( $r$ o $=0.629, p=0.001$ ) as well as between initial mean diastolic blood pressure and ONSDM ( $r h o=0.561, p=0.001)$ in all study groups. Statistically significant differences were determined between the mean pre- and post-treatment systolic and diastolic blood pressures as well as the mean pre- and post-treatment ONSDM values of 40 patients who received antihypertensive therapy $(p=0.000, p=0.000, p=0.000$, respectively).

Conclusion: Ultrasonographic ONSDM reflected increased intracranial pressure in hypertensive patients. The reduction of intracranial pressure was also detected by the same method following antihypertensive treatment. Ultrasonographic ONSDM along with clinical findings and blood pressure measurements may be used for evaluation of response to treatment and deciding on further imaging.
\end{abstract}

Keywords: Hypertension, intracranial pressure, optic nerve sheath diameter, ultrasonography

\section{Introduction}

Hypertension is a health problem for preventive medicine with a gradually increasing importance. Prevalence of hypertension increases with age. Hypertension is detected more than half of people between 60 and 69 years of age and in $3 / 4$ of people at the age of 70 years or older (1). Approximately one billion people are predicted to be hypertensive worldwide, and almost seven million people die due to hypertension every year (2). The rate of annual hospitalization related to hypertensive emergencies is 1-2 per million, and this rate appears to be more frequent in developing countries (3). Zampaglione et al. (4) ranked the most common referral complaints of patients with hypertensive emergencies as chest pain (27\%), dyspnea (22\%) and neurological disorders (2\%).

Cite this article as: Dikmetaş C, Ergin M, Savaș Duman Ç, Gülpembe M, Acar T, Yavuz K, Cander B, Girișgin S, Koçak S. Measurement of Optic Nerve Sheath Diameter to Detect Increased Intracranial Pressure in Hypertensive Patients. Eurasian J Emerg Med. 2020;19(1):40-5

(C) Copyright 2020 by the Emergency Medicine Physicians' Association of Turkey Eurasian Journal of Emergency Medicine published by Galenos Publishing House. 
Hypertension may cause an increase in intracranial pressure (ICP). Such an increase is generally detected at a blood pressure over $180 / 120 \mathrm{mmHg}$ (5). The sensitivity of fundoscopic examination performed for pupil edema in physical examination and used to detect acute ICP increase varies between 20\% and 30\% and depends on the attainment of the clinician (6,7). Papilledema may appear several hours after ICP increase (6). The measurement of optic nerve sheath diameter (ONSDM) by ultrasonography (US) is a non-invasive and useful method for the measurement of increased ICP (8).

In the present study, ONSDM by ultrasound was performed to detect ICP increase in hypertensive patients who were referred to ER to investigate whether the optic nerve sheath diameter (ONSD) increased in the presence of hypertension and whether ONSD was altered following antihypertensive treatment.

\section{Materials and Methods}

Male and female patients over 18 years of age who were referred to the Emergency Room of Emergency Medicine, Department of Necmettin Erbakan University Faculty of Medicine between May, 1 15, 2012 and January, $1^{\text {st }}, 2013$ due to high blood pressure (systolic blood pressure $\geq 140 \mathrm{~mm} \mathrm{Hg}$ and/or diastolic blood pressure $\geq 90 \mathrm{~mm} \mathrm{Hg}$ ) were enrolled in the study. Informed consent was obtained from all patients participating in the study.

Hypertension-induced symptoms were determined as headache, double vision, blurry vision, dyspnea, chest pain, dizziness, dullness, nausea, abdominal pain, pain or weakness on extremities, nasal bleeding and confusion in the present study. Patients included in the study were investigated for these symptoms, and they were divided into two groups as "hypertensive-symptomatic" and "hypertensive-asymptomatic".

Healthy volunteers (normotensive and asymptomatic) without any complaints whose blood pressures were below specified limits and whose consents for participation were obtained constituted the control group.

Young patients, patients with incarceration, those who did not give consent, patients who were unstable and required urgent medical therapy, and symptomatic patients with normal blood pressure were excluded from the study.

Blood pressures of the patients and the volunteers enrolled into the study were measured from the right and left upper extremities following at least 5 minutes of rest by using a sphygmomanometer with an appropriately sized sleeve which was calibrated every year, and the results were recorded on the study form. The blood pressure measurements of the patients in the hypertensive-symptomatic group were repeated at the 30th minute following antihypertensive therapy, and the results were also recorded on the study form. The arithmetic means of recorded systolic and diastolic blood pressures measured from both extremities were calculated for statistical analysis.

ONSDM was performed on axial and sagittal planes from both eyes by a Philips Envisorc brand ultrasound device (serial number: US60608496) using a 7.5 MHz linear probe. Researchers carrying out the study were selected from physicians who were certificated by basic US courses. US imaging was performed in the supine position. Eyelids of the patients were irrigated with water while the eyes were closed. The gel was applied to the US probe. A protective sheath was placed onto the probe to prevent any possible allergic reaction against the gel. The image was saved when the optic nerve sheath image was captured. The distance of $0.3 \mathrm{~cm}$ posterior to the globe was marked, and the distance between both hypoechoic areas surrounding the optic nerve sheath at this plane was measured. Such measurements were applied to both eyes on the sagittal and axial planes. The measurement results were recorded in the study form.

Blood pressure measurements of the patients in the symptomatic hypertensive group were repeated at the $30^{\text {th }}$ minute following antihypertensive therapy. The average of four measurements was calculated to be used for statistical analysis.

The study included a total of 149 participants, including 54 patients in the hypertensive-symptomatic group, 45 patients in the hypertensive-asymptomatic group, and 50 individuals in normotensive- asymptomatic (control) group.

\section{Statistical Analysis}

SPSS ${ }^{T M} 16.0$ version program was used for statistical analysis of the data obtained. The chi-square test was utilized for categorical data. Normality analysis was performed for numeric data. The Wilcoxon sign test was utilized for dependent groups, whereas the Kruskal-Wallis test was used for more than two independent groups, which did not fit a normal distribution. When needed, the Mann-Whitney U test was performed for two independent groups which did not fit a normal distribution. Pearson's and Spearman's correlation analysis were applied to numeric data. For all tests, $p$ values below 0.05 were considered as statistically significant.

\section{Results}

One hundred and forty-nine participants, including 77 (51.7\%) females and 72 (48.3\%) males were enrolled in the study. The control group consisted of 50 healthy volunteers, including 26 (52\%) males and 24 (48\%) females. The hypertensiveasymptomatic group consisted of 45 patients, including 27 (60\%) 
females and 18 (40\%) males. The hypertensive-symptomatic group consisted of 54 patients, including 28 (51.9\%) males and 26 (48.1\%) females (Table 1). There were no statistically significant differences among the study groups regarding gender $(p=0.409)$.

The average age was $27.76 \pm 5.77$ years in the control group, whereas it was $65.11 \pm 15.96$ years in the hypertensiveasymptomatic group and $64.98 \pm 13.06$ years in the hypertensivesymptomatic group (Table 2). A statistically significant difference was determined between the control group and hypertensive-asymptomatic group as well as the control group and hypertensive-symptomatic group ( $p=0.000, p=0.000$, respectively) regarding age. However, there was no statistically significant difference regarding age between the hypertensiveasymptomatic group and hypertensive-symptomatic group $(p=0.816)$. This result was referred to the fact that the control group consisted of normotensive and asymptomatic healthy volunteers.

The mean of initial systolic blood pressure measurements in ER was found as $111.70 \pm 9.77 \mathrm{mmHg}$ in the control group, $165.66 \pm 18.19 \mathrm{mmHg}$ in the hypertensive-asymptomatic group,

Table 1. Distribution of the study groups according to the gender

\begin{tabular}{|l|l|l|l|}
\hline \multirow{2}{*}{ Study Group } & \multicolumn{2}{|l|}{ Gender } & \multirow{2}{*}{ Total } \\
\cline { 2 - 3 } & $\begin{array}{l}\text { Male } \\
\mathbf{n}(\%)\end{array}$ & $\begin{array}{l}\text { Female } \\
\mathbf{n}(\%)\end{array}$ & $\mathbf{n}(\%)$ \\
\hline Normotensive-asymptomatic & $26(52 \%)$ & $24(48 \%)$ & $50(100 \%)$ \\
\hline Hypertensive-asymptomatic & $18(40 \%)$ & $27(60 \%)$ & $45(100 \%)$ \\
\hline Hypertensive-symptomatic & $28(52 \%)$ & $26(48 \%)$ & $54(100 \%)$ \\
\hline Total & $72(48 \%)$ & $77(52 \%)$ & $149(100 \%)$ \\
\hline
\end{tabular}

Table 2. Mean, median, minimum, and maximum values of the study groups regarding age

\begin{tabular}{|l|l|l|}
\hline \multirow{2}{*}{ Study group } & \multicolumn{2}{|l|}{ Age (years) } \\
\cline { 2 - 3 } & Mean \pm SD & Median (min-max) \\
\hline Normotensive-asymptomatic & $27.76 \pm 5.77$ & $26(19-49)$ \\
\hline Hypertensive-asymptomatic & $65.11 \pm 15.96$ & $68(18-97)$ \\
\hline Hypertensive-symptomatic & $64.98 \pm 13.06$ & $68(19-85)$ \\
\hline SD: Standard deviation, min: Minimum, max: Maximum \\
\hline
\end{tabular}

and $177.12 \pm 22.58 \mathrm{mmHg}$ in the hypertensive-symptomatic group (Table 3). In the comparison of the study groups regarding the mean initial systolic blood pressure, statistically significant differences were determined between the control group and the hypertensive-asymptomatic group as well as the control group and hypertensive-symptomatic group ( $p=0.000, p=0.000$, respectively). Furthermore, a statistically significant difference was found to be present between the hypertensive-asymptomatic group and hypertensive-symptomatic group regarding the initially measured mean systolic blood pressure $(p=0.009)$ (Table $3)$.

The mean initial diastolic blood pressure in ER was $76.35 \pm 7.71$ $\mathrm{mmHg}$ in the control group, $93.66 \pm 10.73 \mathrm{mmHg}$ in the hypertensive-asymptomatic group and $100.32 \pm 11.79 \mathrm{mmHg}$ in the hypertensive-symptomatic group (Table 3 ). In the comparison of the study groups regarding the mean initial diastolic blood pressure, statistically significant differences were determined between the control group and hypertensive-asymptomatic group as well as the control group and hypertensive-symptomatic group $(p=0.000, p=0.000$, respectively). Furthermore, a statistically significant difference was found to be present between the hypertensive-asymptomatic and hypertensive-symptomatic groups $(p=0.005)$.

The mean of initial ONSDM in ER was $0.442 \pm 0.0386 \mathrm{~cm}$ in the control group, $0.523 \pm 0.0570 \mathrm{~cm}$ in the hypertensiveasymptomatic group, and $0.527 \pm 0.0652 \mathrm{~cm}$ in the hypertensivesymptomatic group (Table 4). In the comparison of the study groups regarding the initial ONSDM in ER, statistically significant differences were determined to be present between the control group and the hypertensive-asymptomatic group as well as the control group and the hypertensive-symptomatic group ( $p=0.000, \quad p=0.000$, respectively). However, no statistically significant difference was detected between the hypertensiveasymptomatic and hypertensive-symptomatic groups regarding ONSDM $(p=0.254)$.

A statistically significant moderate-to-well correlation was detected between the initial mean systolic blood pressure measured during admission to ER and ONSDM in all study groups (rho=0.629, $\mathrm{p}=0.046$ ). A moderate-to-well correlation

Table 3. The mean, median, minimum, maximum values of systolic blood pressure and diastolic blood pressure of the study groups initially measured in the emergency room

\begin{tabular}{|l|l|l|l|l|}
\hline \multirow{2}{*}{ Study group } & \multicolumn{3}{|l|}{ Initial mean systolic blood pressure $(\mathbf{m m H g})$} & \multicolumn{2}{l|}{ Initial mean diastolic blood pressure (mmHg) } \\
\cline { 2 - 5 } & Mean \pm SD & Median (min-max) & Mean \pm SD & Median (min-max) \\
\hline Normotensive-asymptomatic & $111.70 \pm 9.77$ & $110(90-130)$ & $76.35 \pm 7.71$ & $80(60-90)$ \\
\hline Hypertensive-asymptomatic & $165.66 \pm 18.19$ & $160(145-220)$ & $93.66 \pm 10.73$ & $90(80-130)$ \\
\hline Hypertensive-symptomatic & $177.12 \pm 22.58$ & $175(145-240)$ & $100.32 \pm 11.79$ & $100(75-125)$ \\
\hline SD: Standard deviation, min: Minimum, max: Maximum & \multicolumn{4}{l}{} \\
\hline
\end{tabular}


was detected between the initial mean diastolic blood pressure measured during admission to ER and ONSDM in all study groups, and such correlation was also statistically significant $(\mathrm{rho}=0.561$, $\mathrm{p}=0.000$ ).

A statistically significant correlation was detected between the initial mean systolic blood pressure and ONSDM in both hypertensive groups (hypertensive-asymptomatic and hypertensive-symptomatic) (rho $=0.382, p=0.000$ ). Moreover, a statistically significant correlation was detected between the initial mean diastolic blood pressure and ONSDM $(r h o=0.280$, $\mathrm{p}=0.005)$.

A statistically significant difference was detected between the pre- and post-treatment mean systolic blood pressures of 40 patients in the hypertensive-symptomatic group who received antihypertensive treatment in ER $(p=0.000)$. Similarly, a statistically significant difference was detected between the preand post-treatment mean diastolic blood pressures in the same group $(p=0.000)$.

The mean of ONSDM in the hypertensive-symptomatic group who received antihypertensive treatment in the ER was $0.528 \pm 0.072 \mathrm{~cm}$ before the treatment, and $0.461 \pm 0.058 \mathrm{~cm}$ after the treatment (Table 5). A statistically significant difference was determined between the pre- and post-treatment ONSDM values $(p=0.000)$.

Nineteen patients in the hypertensive-symptomatic group were detected to have at least one of head/brain computed

\begin{tabular}{|c|c|c|}
\hline \multirow[t]{2}{*}{ Study group } & \multicolumn{2}{|c|}{ Initial ONSDM $(\mathrm{cm})$} \\
\hline & Mean \pm SD & \begin{tabular}{|l|} 
Median \\
(min-max)
\end{tabular} \\
\hline Normotensive-asymptomatic & $0.442 \pm 0.0386$ & $0.435(0.371-0.533)$ \\
\hline Hypertensive-asymptomatic & $0.523 \pm 0.0570$ & $0.523(0.389-0.619)$ \\
\hline Hypertensive-symptomatic & $0.527 \pm 0.0652$ & $0.527(0.388-0.706)$ \\
\hline
\end{tabular}

Table 5. Mean, median, minimum, maximum values of ONSDM before and after the treatment of the hypertensivesymptomatic patients

\begin{tabular}{|l|l|l|}
\hline \multirow{2}{*}{ Study group } & \multicolumn{2}{|l|}{ ONSDM $(\mathbf{c m})$} \\
\cline { 2 - 3 } & Mean \pm SD & Median (min-max) \\
\hline Before treatment & $0.528 \pm 0.072$ & $0.532(0.388-0.706)$ \\
\hline After treatment & $0.461 \pm 0.058$ & $0.449(0.361-0.586)$ \\
\hline $\begin{array}{l}\text { ONSDM: Optic nerve sheath diameter measurement, SD: Standard deviation, min: } \\
\text { Minimum, max: Maximum }\end{array}$ \\
\hline
\end{tabular}

tomography (CT), head/brain MR, head/brain CT angiography and/or head/brain MR angiography imaging tests. The evaluation of these tests by the Radiology Department, revealed no acute pathology in five patients. Various pathologies were detected in 14 patients as follows: four patients with intraparenchymal bleeding, 1 patient with subarachnoid bleeding, 1 patient with subdural bleeding, three patients with acute/subacute infarction, three patients with chronic infarction, one patient with meningioma, and one patient with encephalitis. Since encephalitis, meningioma, and chronic infarction were not acute complications of hypertension, they were excluded from the subgroup analysis. Therefore, nine patients with acute pathology were compared with the other five patients regarding ONSDM. The average value of ONSDM was $0.480 \pm 0.044 \mathrm{~cm}$ in the group with no acute pathology and $0.571 \pm 0.057 \mathrm{~cm}$ in the group with an acute pathology (Table 6). A statistically significant difference was found to be present between the two groups regarding ONSDM $(p=0.009)$.

\section{Discussion}

Although ICP measurement by invasive intracranial devices is a gold standard, the measurement is not always possible because the procedure is invasive, requires being specialized in neurosurgery, and has some contraindications such as coagulopathy or thrombocytopenia (9). Non-invasive techniques such as MRI or CT may be preferred; however, they are both expensive and time-consuming (10). Furthermore, CT scanning cannot be performed in unstable patients. Neuroimaging should be performed in a patient with a suspicion of ICP increase to exclude a space-occupying lesion, which requires surgical decompression (11). Moreover, findings supporting the diagnosis of increased ICP include signification of the subarachnoid field around the optic nerve, papilledema (posterior sclera flattening), vertical curvature of the optic nerve, prominent prelaminar part of the optic nerve, partially empty sella turcica, flow reduction in dural venous sinus, and sinus compression $(12,13)$. Transcranial doppler US, which requires experience, might be insufficient (14).

The optic nerve is a part of the central nervous system during development and surrounded by subarachnoid cerebrospinal

Table 6. Mean, median, minimum and maximum values of ONSDM of the hypertensive-symptomatic patients with/ without pathology detected by advanced imaging tests

\begin{tabular}{|l|l|l|}
\hline \multirow{2}{*}{ Study group } & \multicolumn{2}{|l|}{ ONSDM $(\mathbf{c m})$} \\
\cline { 2 - 3 } & Mean \pm SD & Median (min-max) \\
\hline No pathology $(\mathbf{n = 5})$ & $0.480 \pm 0.044$ & $0.487(0.418-0.532)$ \\
\hline Pathology $(\mathbf{n}=\mathbf{9})$ & $0.571 \pm 0.057$ & $0.573(0.514-0.706)$ \\
\hline
\end{tabular}

ONSDM: Optic nerve sheath diameter measurement, SD: Standard deviation, min: Minimum, max: Maximum 
fluid and dura mater; therefore, ONSDM is affected by cerebrospinal fluid changes (15). There are many studies addressing the relation between ONSDM and ICP in the literature. Kimberly et al. (16) analyzed the correlation between ONSDM and direct invasive ICP measurement in their study conducted in 15 individuals (11 individuals with spontaneous intracerebral hemorrhage; 4 individuals with traumatic injury) by optic US. They determined the presence of a significant correlation between ICP and ONSDM. When ONSDM was measured to be larger than $0.5 \mathrm{~cm}$, the sensitivity and specificity were $88 \%$ and $93 \%$, respectively, regarding the detection of ICP increase (16).

Blaivas et al. (17) conducted their study in 35 individuals and detected increased ICP findings in CT scan of 14 patients. Mean ONSDM of the patients with increased ICP and without pathology by CT was measured $6.27 \mathrm{~mm}$ and $4.42 \mathrm{~mm}$, respectively. They reported that they found no statistically significant difference between the two groups. The sensitivity and specificity for detection of ICP increase by ONSDM by US were found to be $95 \%$ and $100 \%$, respectively (17).

Roque et al. (8) measured ONSD by US to detect the increase of ICP in hypertensive patients. In their study, patients with a systolic blood pressure of $\geq 140 \mathrm{mmHg}$ and/or diastolic blood pressure of $\geq 90 \mathrm{mmHg}$ were included in the hypertensive group. The patients in the hypertensive group were divided into two subgroups. A patient was placed in the hypertensivesymptomatic group if he/she had one or more complaints such as headache, double vision, chest pain, shortness of breath, drowsiness, dizziness, nausea/vomiting, abdominal pain, and pain/weakness on the extremities and in the hypertensiveasymptomatic group if no complaint was present. Patients with a systolic blood pressure of $<140 \mathrm{mmHg}$ and/or diastolic blood pressure of $<90 \mathrm{mmHg}$ who did not have any complaints were included in the normotensive-asymptomatic group (8). In line with the present study, Roque et al. (8) detected a correlation between the mean systolic blood pressure and ONSDM when they analyzed all groups. Moreover, a weak correlation was found between the mean diastolic blood pressure and ONSDM when compared with the correlation between systolic blood pressure and ONSDM (8). We detected a moderate-to-well and significant correlation in our study. Similarly, in the study of Roque et al. (8), a correlation was detected between systolic blood pressure and ONSDM in the hypertensive group. Roque et al. (8) also found a correlation between the mean diastolic blood pressure and ONSDM. We detected a significant correlation in the present study. Furthermore, Roque et al. (8) accepted ONSDM $\geq 0.5 \mathrm{~cm}$ as abnormal and detected the best systolic and diastolic blood pressure threshold providing that level as $166 / 82 \mathrm{mmHg}$. In the same study, no statistically significant difference was detected in
ONSDM before and after antihypertensive treatment. However, in the present study, a statistically significant difference was detected in the hypertensive-symptomatic group before and after antihypertensive treatment.

Girisgin et al. (18) enrolled 54 individuals, including 26 healthy volunteers in their study. CT scan reports of the patient group revealed brain injury, intracerebral hemorrhage, cerebral infarction, and hypoxic brain. Mean ONSDM was found as 4.6 $\mathrm{mm}$ in healthy volunteers and $6.4 \mathrm{~mm}$ in the patient group. A statistically significant difference was detected between the two groups (18). A significant difference was detected between the patients with and without acute intracranial pathology by CT within the hypertensive symptomatic group of the present study.

Amini et al. (19) measured ONSDM on 50 patients who were hemodynamically stable and had lumbar puncture because of various causes. ICP measurement was performed by lumbar puncture in that study. ICP was detected to be normal in 36 patients and increased in 14 patients. Mean ONSDM in the patients with increased ICP was found $6.66 \pm 0.58 \mathrm{~mm}$ and mean ONSDM in the patients with a normal ICP was detected $4.60 \pm 0.41$ $\mathrm{mm}$. A statistically significant difference was detected between the two groups $(p<0.001)$. A statistically significant correlation was detected between the ONSDM value and ICP (rho $=0.88$, $\mathrm{p}<0.05$ ). When ONSDM was over $5.5 \mathrm{~mm}$, the sensitivity and specificity regarding ICP over $20 \mathrm{~cm} \mathrm{H} 20$ were $100 \%$ and $100 \%$, respectively (19).

Bäuerle et al. (20) searched the relation between ONSDM and ICP on 25 patients diagnosed with IIH. The control group included 25 healthy volunteers who did not have lumbar puncture before. In the aforementioned that study, ICP was measured by lumbar puncture and 30 to $50 \mathrm{~mL}$ cerebrospinal fluid was discharged as a treatment. After the discharge procedure, ONSDM was performed again. Mean ONSDM in the patients with IHH and in the control group was found $6.4 \pm 0.6 \mathrm{~mm}$ and $5.4 \pm 0.5 \mathrm{~mm}$; and there was statistically significant difference between groups $(p<0.001)$. Moreover, the best threshold of ONSDM to detect ICP increase was found to be $5.8 \mathrm{~mm}$; sensitivity and specificity were $90 \%$ and $84 \%$, respectively. ONSDM decreased on both eyes in the patients with IHH after lumbar puncture (right ONSDM $5.8 \pm 0.7 \mathrm{~mm}$, $p<0.004$; left ONSDM $5.9 \pm 0.7 \mathrm{~mm}(p<0.043)(20)$. The results of the present study and of the studies conducted by Amini et al. (19) have led us to think that the efficacy of treatments for reduction of ICP can be monitored by ultrasonographic ONSDM.

\section{Study Limitations}

In the present study, the control group was created from normotensive-asymptomatic healthy volunteers. The average ages of the control and hypertensive study groups were different. 
Importance should be given to age factor regarding the selection of healthy volunteers in more extensive studies planned in the future.

Researchers did not affect clinical follow-up and progress of the patients enrolled in the observational study, which was performed prospectively. Therefore, the number of advanced imaging studies in the symptomatic hypertensive group was 19. Within the scope of further studies, advanced imaging may be performed in all patients included in the symptomatic hypertensive group on the condition to obtain approval of the ethics committee and consent of the patient.

Complaints expressed by the patients were considered for the differentiation of hypertensive-asymptomatic and hypertensivesymptomatic groups in the present study. Therefore, groups were determined according to the subjective criteria. We believe that this might have contributed to the outcome that the increase of ONSDM is independent of the symptoms. We suggest a review of the relation between symptoms of hypertension and ONSDM in further studies.

\section{Conclusion}

Since ultrasonographic examination does not involve radiation, and it is an applicable, repeatable, and cost-efficient method. Use of US increases in ERs gradually. Measurement of ONSD by US may provide information about ICP increase and may be used for diagnosis and follow-up of patients with suspicion of ICP increase, including hypertensive emergencies. Emergency medicine physicians should learn how to use US in this field along with other fields of use.

\section{Ethics}

Ethics Committee Approval: This prospective clinical study was approved by the Local Ethics Committee at Necmettin Erbakan University Meram Medicine Faculty (date: 13/04/2012 - no: 2012/76) and conducted at Faculty of Medicine Hospital Emergency Department.

Informed Consent: Informed consent was obtained from all patients participating in the study.

Peer-review: Externally peer-reviewed.

\section{Authorship Contributions}

Concept: C.D., M.E., Ç.S.D., M.G., T.A., K.Y., B.C., S.G., S.K., Design: C.D., M.E., Ç.S.D., M.G., T.A., K.Y., B.C., S.G., S.K., Data Collection or Processing: C.D., M.E., Ç.S.D., M.G., T.A., K.Y., B.C., S.G., S.K., Analysis or Interpretation: C.D., M.E., Ç.S.D., M.G., T.A., K.Y., B.C., S.G., S.K., Literature Search: C.D., M.E., C..S.D., M.G., T.A., K.Y., B.C., S.G., S.K., Writing: C.D., M.E., Ç.S.D., M.G., T.A., K.Y., B.C., S.G., S.K.
Conflict of Interest: No conflict of interest was declared by the authors.

Financial Disclosure: The authors declared that this study received no financial support.

\section{References}

1. Burt VL, Whelton P, Roccella EJ, Brown C, Cutler JA, Higgins M, et al. Prevalence of hypertension in the US adult population. Results from the Third National Health and Nutrition Examination Survey, 1988-1991. Hypertension. 1995;25:305-13.

2. Organization WH. The world health report 2002: reducing risks, promoting healthy life. In: Organization WH, editor. 2002.

3. Varon J, Strickman NE. Diagnosis and treatment of hypertensive crises in the elderly patients. J Geriatr Cardiol. 2007;4:50-5.

4. Zampaglione B, Pascale C, Marchisio M, Cavallo-Perin P. Hypertensive urgencies and emergencies. Prevalence and clinical presentation. Hypertension. 1996;27:144-7.

5. Tintinalli's Emergency Medicine Seventh Edition: A Comprehensive Study Guide. Tintinalli JE, Stapcyznski JS, Ma OJ, Cline DM, Cydulka RK, Mecker GD, editors. New York: McGraw Medical; 2011.

6. Geeraerts T, Launey Y, Martin L, Pottecher J, Vigue B, Duranteau J, et al. Ultrasonography of the optic nerve sheath may be useful for detecting raised intracranial pressure after severe brain injury. Intensive Care Med. 2007;33:170411.

7. Trobe JD. Papilledema: the vexing issues. J Neuroophthalmol. 2011;31:175-86.

8. Roque PJ, Wu TS, Barth L, Drachman D, Khor KN, Lovecchio F, et al. Optic nerve ultrasound for the detection of elevated intracranial pressure in the hypertensive patient. Am J Emerg Med. 2012;30:1357-63.

9. Rickert K, Sinson G. Intracranial pressure monitoring. Operative Techniques in General Surgery. 2003;5:170-5.

10. Dubourg J, Javouhey E, Geeraerts T, Messerer M, Kassai B. Ultrasonography of optic nerve sheath diameter for detection of raised intracranial pressure: a systematic review and meta-analysis. Intensive Care Med. 2011;37:1059-68.

11. Emmez ÖH, Egemen E. Kafa içi basınç artışı tedavisinde pratik yaklaşımlar. Yoğun Bakım Dergisi. 2010;9:77-84.

12. Zagardo MT, Cail WS, Kelman SE, Rothman MI. Reversible empty sella in idiopathic intracranial hypertension: an indicator of successful therapy? AJNR Am J Neuroradiol. 1996;17:1953-6.

13. Suzuki H, Takanashi J, Kobayashi K, Nagasawa K, Tashima K, Kohno Y. MR imaging of idiopathic intracranial hypertension. AJNR Am J Neuroradiol. 2001;22:196-9.

14. Aaslid R, Huber P, Nornes H. A transcranial Doppler method in the evaluation of cerebrovascular spasm. Neuroradiology. 1986;28:11-6.

15. Hayreh SS. Pathogenesis of Oedema of the Optic Disc (Papilloedema). A Preliminary Report. Br J Ophthalmol. 1964;48:522-43.

16. Kimberly HH, Shah S, Marill K, Noble V. Correlation of optic nerve sheath diameter with direct measurement of intracranial pressure. Acad Emerg Med. 2008;15:201-4.

17. Blaivas M, Theodoro D, Sierzenski PR. Elevated intracranial pressure detected by bedside emergency ultrasonography of the optic nerve sheath. Acad Emerg Med. 2003;10:376-81.

18. Girisgin AS, Kalkan E, Kocak S, Cander B, Gul M, Semiz M. The role of optic nerve ultrasonography in the diagnosis of elevated intracranial pressure. Emerg Med J. 2007;24:251-4.

19. Amini A, Kariman H, Arhami Dolatabadi A, Hatamabadi HR, Derakhshanfar $\mathrm{H}$, Mansouri B, et al. Use of the sonographic diameter of optic nerve sheath to estimate intracranial pressure. Am J Emerg Med. 2013;31:236-9.

20. Bäuerle J, Nedelmann M. Sonographic assessment of the optic nerve sheath in idiopathic intracranial hypertension. J Neurol. 2011;258:2014-9. 